\title{
A EVASÃO NO CURSO DE ENGENHARIA DE ELÉTRICA E ANÁLISE DE UM ACOMPANHAMENTO COMPLEMENTAR EM DISCIPLINAS DE CÁLCULO E CIRCUITOS ELÉTRICOS
}

DOI: 10.37702/2175-957X.COBENGE.2021.3680

Ana Paula Chaves Cabral - ana.cabral@cear.ufpb.br

Universidade Federal da Paraíba

Rua José Sinfrônio de Oliveira Mariz 101

58073-203 - João Pessoa - PB

Maria Heloisa de Pietra Sllva - maria.silva@cear.ufpb.br

Universidade Federal da Paraíba

Avenida Major Augusto Bezerra 510

58228-000 - Dona Inês - PB

Emmanuela Tertuliano Moreira de Sousa - emmanuela.sousa@cear.ufpb.br Universidade Federal da Paraíba

Av. Comandante Matos Cardoso 140

58050-120 - João Pessoa - PB

Elton Davi Ramos da Silva - elton.silva@cear.ufpb.br

Universidade Federal da Paraíba

Rua 7 de setembro 10

58250-000 - Lagoa de Dentro - PB

Euler Cássio Tavares de Macedo - euler@cear.ufpb.br

Universidade Federal da Paraíba

Rua Vandik Pinto Figueiras 158

58042-110 - João Pessoa - PB

Resumo: Devido aos números relativamente altos dos índices de evasão e de reprovação nas disciplinas que compõem a grade curricular inicial do curso de Engenharia Elétrica da Universidade Federal da Paraíba, urge a necessidade da elaboração de materiais e métodos que minimizem esses números. Dessa forma, neste trabalho um grupo de estudantes da UFPB busca analisar os impactos referentes aos cursos de Cálculo I e Circuitos elétricos I que foram elaborados pelo 


\section{COBENCE - Evento online

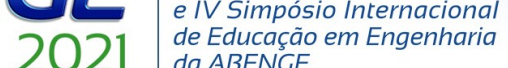 \\ 28 a 30 de SETEMBRO \\ "Formação em Engenharia: \\ Tecnologia, Inovaçâo e Sustentabilidade"}

grupo como forma de acompanhamento complementar nessas disciplinas.

Palavras-chave: Evasão. Graduação. Engenharia. 
 \\ A EVASÃO NO CURSO DE ENGENHARIA DE ELÉTRICA E ANÁLISE DE UM ACOMPANHAMENTO COMPLEMENTAR EM DISCIPLINAS DE CÁLCULO E CIRCUITOS ELÉTRICOS}

\section{INTRODUÇÃO}

No Brasil, a partir do início do século XXI, o governo federal foi responsável por um maior investimento na educação superior pública, e o número de matrículas em instituições de ensino superior públicas passou de 944.584 no ano de 2001, chegando a 2.080.146 no ano de 2019 (INEP, 2010; INEP 2019).

Contudo, assim como é grande o número de matrículas na graduação e de programas do governo como o Programa Nacional de Assistência Estudantil, que fornece apoio à permanência aos estudantes de baixa renda, a evasão no ensino superior é também bastante expressiva. Estima-se que no mundo a evasão no primeiro ano de curso é entre duas a três vezes mais alta que nos anos subsequentes, isso torna-se um problema para as instituições de ensino devido ao desperdício de tempo, recursos acadêmicos e econômicos utilizados, além de provocar frustração nos jovens estudantes em seus objetivos de formação (SILVA FILHO et al, 2007).

Dessa forma, é importante proporcionar um apoio aos ingressantes, como meio de nivelamento, principalmente aos que ingressaram no âmbito da pandemia da COVID-19 com o ensino remoto. As atividades de nivelamento têm um papel fundamental para resgatar a democratização do ensino, facilitando a permanência do aluno na universidade, resgatando a apropriação de conhecimentos esquecidos ou não apreendidos, facilitando a otimização do processo de ensino aprendizagem (BARRETO et al, 2011).

Normalmente, o curso de Engenharia Elétrica possui disciplinas gerais de matemática e física, disciplinas profissionais básicas, tais como circuitos elétricos, eletromagnetismo, eletrônica e disciplinas de formação profissional específica que depende da ênfase de formação dos alunos. Bossa (2019) e Cristina e Bispo (2017) apontam que as disciplinas gerais de matemática e física do ciclo básico geram grandes dificuldades para alunos com pouca base nesses conteúdos, sendo assim um obstáculo na adaptação desses alunos no ensino superior e até contribuindo na desistência dos ingressantes nos primeiros anos de curso.

Visto isso, o grupo PET-Elétrica da Universidade Federal da Paraíba (UFPB), com apoio da assessoria de graduação do Centro Energias Alternativas e Renováveis (CEAR), analisou os maiores índices de reprovação em componentes curriculares do curso de graduação em Engenharia Elétrica da UFPB, conforme apresentado na Figura 1. Com o intuito de reduzir as taxas de reprovação nas duas disciplinas com maior índice de reprovação, sendo essas Circuitos Elétricos I e Cálculo Diferencial e Integral I, o PETElétrica elaborou dois cursos on-line com resolução de exercícios para essas duas disciplinas. 
Figura 1 - Distribuição das disciplinas com maiores taxas de reprovação.

Disciplinas com maiores taxas de reprovação no curso de Engenharia Elétrica da UFPB

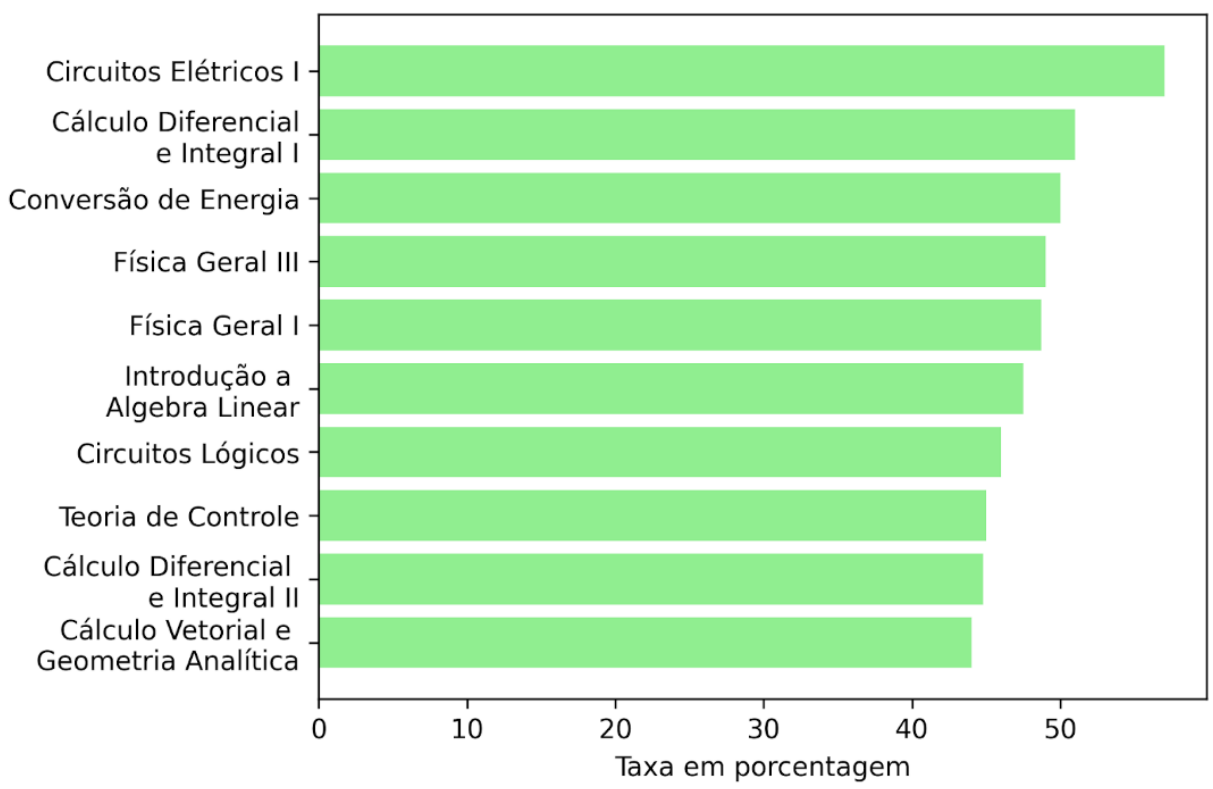

Fonte: Centro de Energias Alternativas e Renováveis da UFPB.

Neste artigo é apresentado como os cursos foram desenvolvidos e os resultados obtidos. Devido ao cenário pandêmico, foi necessário realizar a adaptação do conteúdo ao ensino remoto e, para isso, foram elaborados vídeos nos quais as aulas tiveram como foco a resolução de exercícios contextualizados e posteriormente divulgados no canal do Youtube "PET Elétrica UFPB Minicursos" com principal objetivo de motivar e complementar os estudos dos acadêmicos. Assim, minimizar a carência de conteúdos base do curso de Engenharia Elétrica e aumentar a eficiência do ensino para, desse modo, ajudar a mitigar a evasão ocasionada pela dificuldade nestas disciplinas nos primeiros períodos da graduação.

\section{FUNDAMENTAÇÃO TEÓRICA}

Segundo o Instituto Lobo (2012), algumas das causas mais comuns da evasão do sistema, aquela que o estudante abandona o sistema de ensino, são:

"A baixa qualidade da Educação Básica brasileira: que pode ser mensurada pelos exames internacionais aplicados e é largamente anunciada e discutida, com ênfase cada vez maior, nos mais diferentes segmentos da sociedade brasileira;

A baixa eficiência e o diploma do Ensino Médio: que não garante a suficiência de competências do candidato ao Ensino Superior, criando dificuldades de adaptação e acompanhamento do curso;"

Observa-se que devido a esses fatores, há uma dificuldade na transição da educação básica para o ensino superior, também pela insuficiência de competências em conteúdos e habilidades que deveriam ter sido bem trabalhadas.

Ademais, com as mudanças na metodologia de ensino trazidas em decorrência do contexto da pandemia, a habilidade de elaborar materiais didáticos audiovisuais recebeu ainda mais destaque, entretanto, segundo Jesus e Solto (2018) uma grande parcela de professores ainda são considerados imigrantes digitais, por não nascerem inseridos na 
época tecnológica, com isso possuem dificuldade de produzir conteúdo pedagógico por meio de ferramentas tecnológicas.

De acordo com Bleicher (2009), 40\% das pessoas respondem melhor a estímulos visuais. Pode-se observar também que, ao ingressar no curso de graduação, é comum uma busca por videoaulas para material de estudo suplementar. Essas buscas ocorrem principalmente, quando o estudante se depara com as disciplinas com muitos pré-requisitos de conteúdos que os alunos possuem maiores dificuldades, que normalmente causam retenção e podem acarretar na desistência do curso. Nesse sentido, é possível observar a importância de elaborar atividades de nivelamento para os ingressantes utilizando os ambientes digitais, pela facilidade em acessar os conteúdos quando necessário.

Sendo assim, dado ambiente de ensino remoto, e buscando facilitar o processo de entendimento de algumas dessas disciplinas, o grupo PET-Elétrica elaborou videoaulas para um acompanhamento suplementar nas disciplinas de Cálculo Diferencial e Integral I e Circuito Elétricos I.

\section{METODOLOGIA}

No ambiente de ensino remoto atual e devido ao cenário pandêmico, buscando contribuir para um melhor processo de aprendizado, o grupo PET-Elétrica disponibilizou videoaulas de resolução de exercícios das disciplinas de Cálculo Diferencial e Integral I e Circuitos Elétricos I. Assim, é possível o compartilhamento de conhecimentos básicos da grade curricular do curso de Engenharia Elétrica de forma aberta para todos que desejarem acessar.

Para a preparação dos vídeos de ambos os minicursos, foi adotada a seguinte metodologia seguindo um processo de etapas cronológicas. Após a decisão dos cursos que seriam realizados por critério de retenção, a primeira etapa é de elaboração do plano de curso baseado na ementa de cada disciplina, para realizar exercícios que cubram os conteúdos de forma satisfatória. O plano de curso elaborado pelos estudantes é revisado por ao menos um professor que ministra a disciplina para ser aprovado e para que sejam feitas as devidas correções.

A preparação das aulas inicia-se com a criação de um roteiro detalhado do que será abordado em cada aula e de ferramentas visuais como os slides com textos, figuras e todo o processo de cálculo das resoluções para tornar as informações mais sucintas e visualmente compreensíveis. Assim que terminado e revisado entre o grupo, começa a gravação das aulas, gravando a tela e áudio pelo software aberto OBS Studio e ferramentas de gravação de áudio nativas de celulares. Com as devidas gravações, os próprios alunos utilizam softwares de edição de vídeo gratuitos e de software aberto como o Openshot e Shotcut para cortes nos vídeos, retirando possíveis erros e pausas demoradas dos arquivos brutos para uma explicação mais fluida, ajustando a qualidade e volume do áudio e deixando os vídeos menores para captar o máximo da atenção do espectador.

As aulas prontas e revisadas foram disponibilizadas semanalmente na plataforma do YouTube, por ser amplamente utilizada na visualização de vídeos, ter um retorno direto com comentários e fornecer métricas acerca do alcance do conteúdo para tornar possível uma avaliação de desempenho do curso e dos alunos.

\subsection{Curso de Cálculo I}

A disciplina de Cálculo Diferencial e Integral I é ministrada no primeiro período no curso de graduação em Engenharia Elétrica da UFPB e faz parte do grupo de disciplinas de cálculo que compõe a grade inicial do curso, visando promover uma oportunidade de nivelamento de conteúdos de matemática aos alunos ingressantes nos cursos de engenharia. 
O curso foi dividido em duas partes: pré-cálculo e cálculo diferencial, que totalizaram 23 videoaulas produzidas. A primeira parte, reunindo os conteúdos mais necessários do ensino médio para serem revisados, assuntos que envolvem desde conjuntos numéricos e intervalos até funções elementares. Esses conteúdos foram disponibilizados nos 6 vídeos iniciais, mostrando a resolução de 11 exercícios em busca de facilitar a compreensão do conteúdo que será visto posteriormente.

Na segunda parte, o curso adentrou no conteúdo de cálculo em si, contendo um total de 17 vídeos, começando por limites e teoremas, e por fim o conteúdo de derivada e outros teoremas. Essa parte do curso contou com questões contextualizadas envolvendo conteúdos como: o uso de função de custo benefício de uma fábrica ao eliminar dióxido de enxofre, introduzindo o conceito de limites e continuidade, derivadas, máximos e mínimos, equação da reta tangente, teorema do valor médio e a regra de L'Hôpital.

O material foi elaborado por meio de videoaulas expositivas de revisão com média de 12 minutos de duração. Foram utilizados slides constituídos por: uma parte teórica referente ao assunto a ser abordado, exemplificações e exercícios contextualizados utilizando recursos como gráficos e figuras. As aulas foram produzidas e ministradas remotamente por integrantes do grupo, sendo uma parte destes responsáveis pela montagem do roteiro, slides, gravação das aulas, e outra parte pela edição e lançamento dos vídeos na plataforma YouTube em 2 aulas por semana durante o período de 22 de junho a 12 de agosto de 2020.

\subsection{Curso de Circuitos Elétricos I}

A disciplina de Circuitos Elétricos I está presente no quarto período da grade curricular da graduação em Engenharia Elétrica, sendo uma disciplina importante para encerrar a base do curso e também a com o maior índice de reprovação. Muitos alunos encontram dificuldades devido a demanda de conhecimento prévio de alguns conceitos básicos dados desde ensino médio na física e matemática aos conhecimentos das disciplinas de física e cálculo dos três períodos iniciais da graduação.

Com o objetivo de auxiliar os alunos que estavam cursando a disciplina de Circuitos Elétricos I durante o período letivo 2020.1 e os períodos subsequentes, o curso foi preparado com o auxílio do professor responsável por ministrá-la.

O processo de criação de roteiro, slides, gravação, edição e divulgação foi análogo ao curso de Cálculo I. No total, foram disponibilizadas 19 aulas que focaram na aplicação do que era ministrado semanalmente nas aulas com exercícios selecionados e resolvidos pelos integrantes do grupo com recursos visuais ilustrados para tornar a resolução mais didática e abordando os conteúdos da disciplina, desde as leis de Kirchhoff, medidores de corrente e tensão, até assuntos mais específicos e avançados, como circuitos de segunda ordem e a transformada de Laplace. As aulas foram postadas semanalmente na plataforma do YouTube acompanhando o decorrer do semestre.

\section{RESULTADOS}

No curso de Cálculo I, ao utilizar da métrica disponibilizada pelo Youtube, pôde-se observar que foi elaborado 5 horas de conteúdo no total, com 471 curtidas e 55 comentários, sendo também possível estabelecer parâmetros a respeito da quantidade de visualizações em cada aula, como mostra a Figura 2, na qual foi obtido uma média de 89 visualizações por vídeo, totalizando 2088 visualizações até o momento em que esse texto foi

escrito.

No gráfico da Figura 2 também é notório que há uma quantidade maior de visualizações logo na primeira aula em comparação com os outros vídeos, referente ao conteúdo de pré cálculo sobre desigualdades, mais estudantes assistiram tendo em vista a 
divulgação dessa aula na estreia do lançamento do canal e do primeiro curso a ser disponibilizado pelo YouTube elaborado pelo grupo.

Figura 2 - Distribuição das visualizações por aula de Cálculo I.

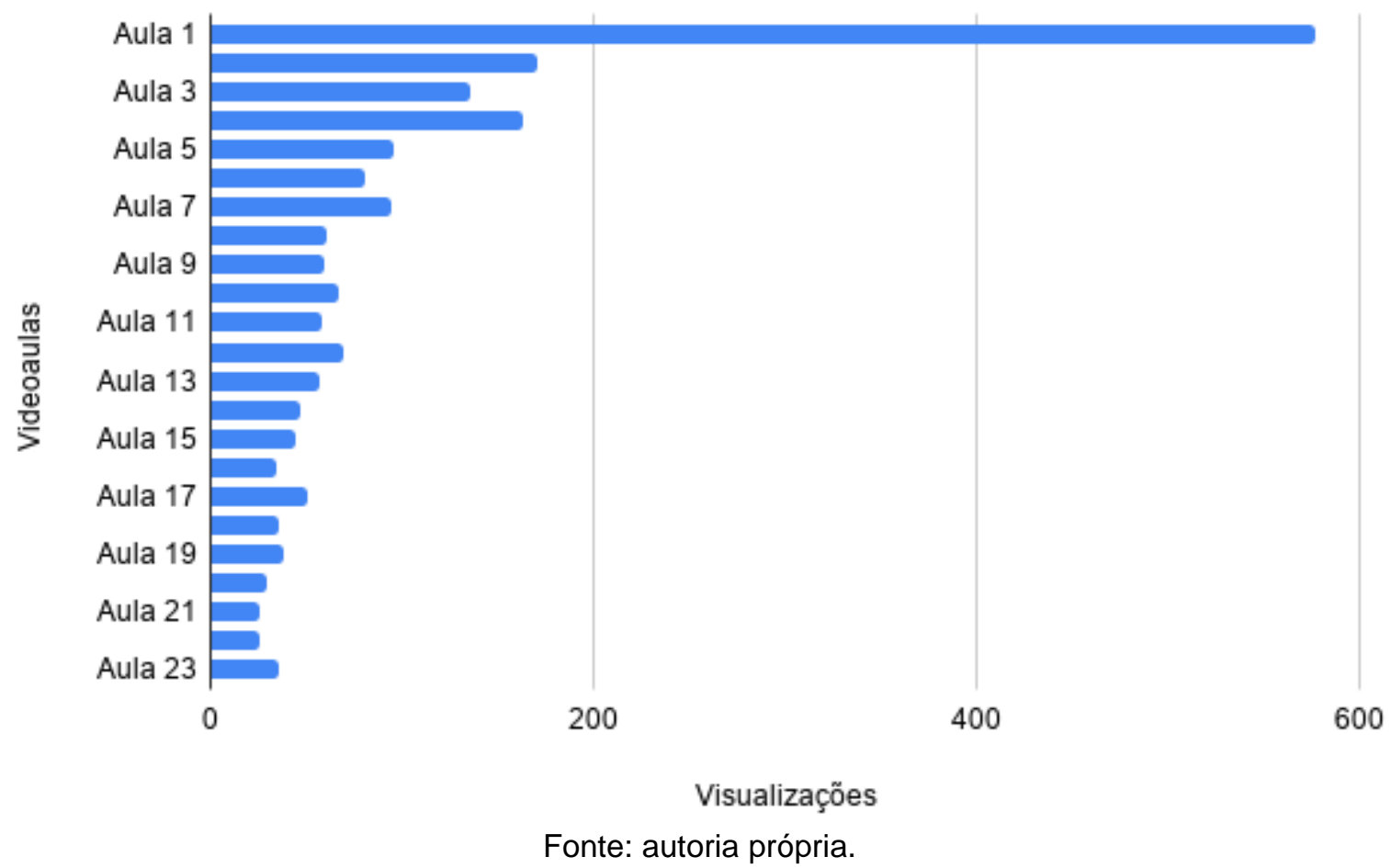

Como forma de agregar na fixação do conteúdo, também foram disponibilizados formulários Google nas descrições dos vídeos com exercícios a serem respondidos, obtendo os dados representados na Figura 3 . No total, foram 16 respostas nas quais foi observado que, dentre os alunos que responderam, alguns eram discentes de universidades públicas e uma privada de outros estados além da Paraíba, como Maranhão e São Paulo, ultrapassando as expectativas ao atingir público externo à Universidade Federal da Paraíba, bem como, outros cursos além de Engenharia Elétrica.

O alcance do curso de Cálculo I demonstra que a dificuldade na disciplina abrange diversos cursos, fator consequente do déficit em matemática básica marcante desde o ensino médio, como mostra os dados do Sistema de Avaliação da Educação Básica (Saeb) feita em 2017, em que o MEC classifica os níveis de proficiência, ao qual no $3^{\circ}$ ano do ensino médio, $71,67 \%$ dos alunos têm nível insuficiente de aprendizado em matemática. 
Figura 3 - Quantidade de respostas nos formulários do curso de Cálculo I.

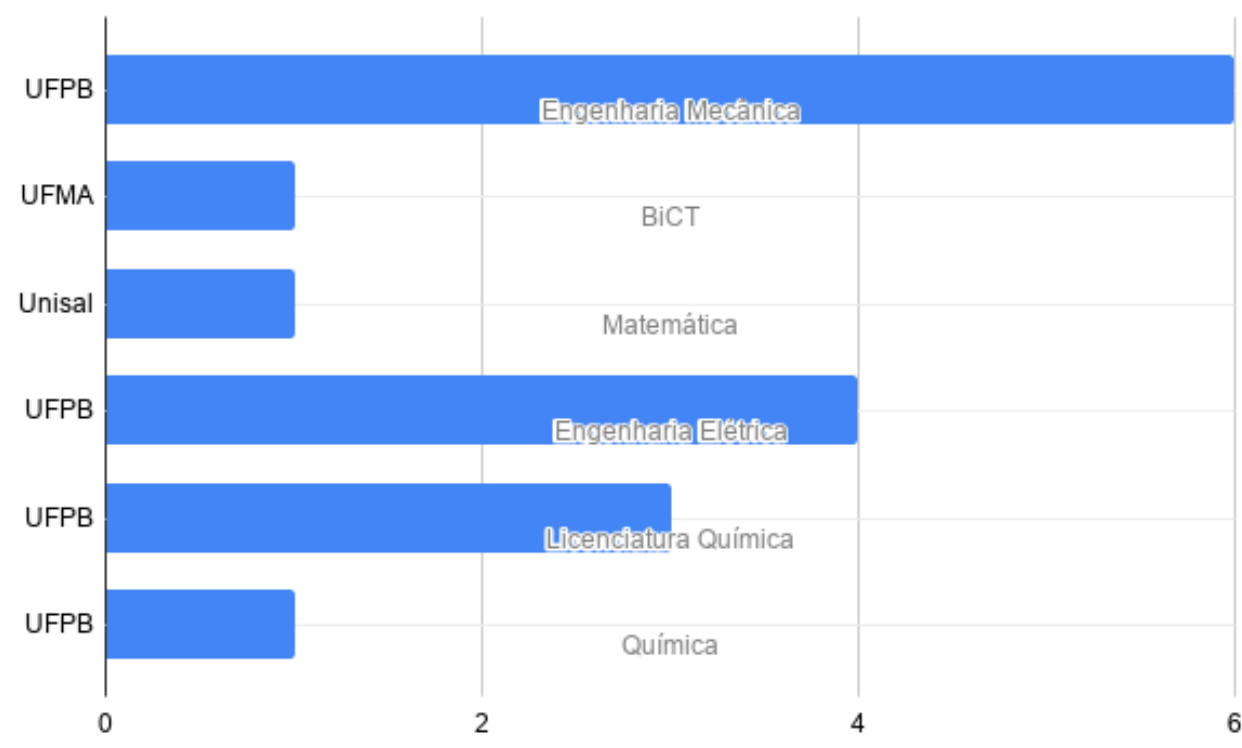

Fonte: autoria própria.

No curso de Circuitos Elétricos I, com um total de 19 aulas gravadas, é possível observar com as métricas visuais do YouTube, um apanhado de 263 curtidas, 25 comentários, 50 horas totais assistidas e 1734 visualizações ao todo. Destas aulas podemse observar na Figura 4 a distribuição das visualizações por cada vídeo. De acordo com o gráfico, é possível obter uma média de 91 visualizações por aula.

Figura 4 - Distribuição das visualizações por aula de Circuitos Elétricos I.

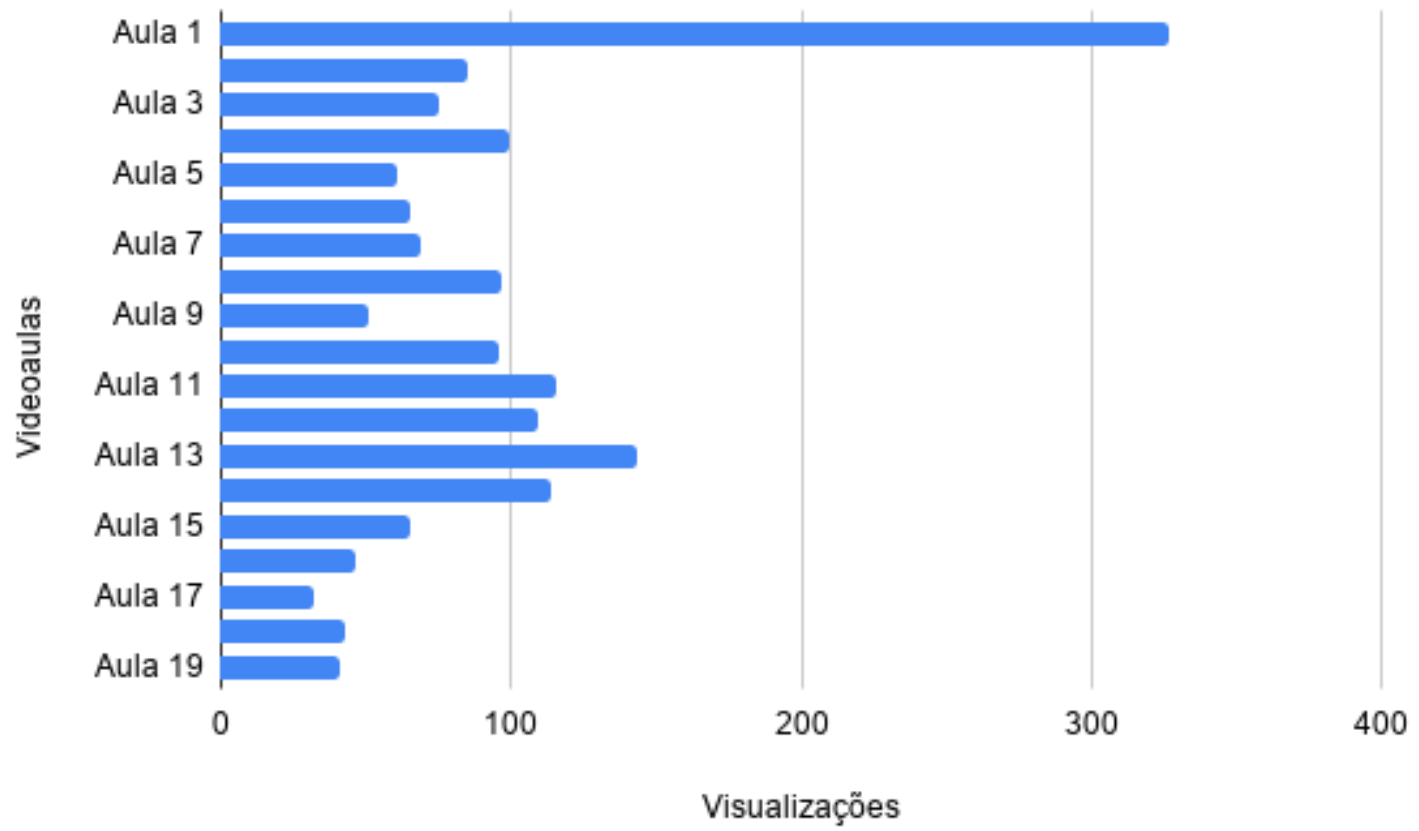

Fonte: autoria própria.

Diferente da disciplina de Cálculo I, Circuitos Elétricos I é específica da grade curricular do curso de Engenharia Elétrica, o que ocasionou um público um pouco mais restrito e menos visualizações em comparação com a primeira. Porém, é perceptível na 
Figura 2 um desvio padrão maior do que na Figura 4 com a discrepância entre o número de visualizações, tornando os dados não tão uniformes. A disciplina de Cálculo I possui um público maior que não abrange apenas cursos de engenharia e possui mais material disponível na internet. Já a disciplina de Circuitos Elétricos I tem um público restrito e menos conteúdo disponibilizado na internet, sendo mais fácil pesquisar determinado assunto específico e encontrar os vídeos que foram disponibilizados pelo grupo PET-Elétrica.

Além disso, analisando a distribuição da quantidade de aulas de ambos os cursos, os vídeos de Circuitos Elétricos I possuem uma média de visualizações maior em comparação com o curso de Cálculo I, tornando evidente que a procura pelas aulas de Circuitos Elétricos I, e um desvio padrão menor durante todo o semestre.

\section{CONSIDERAÇÕES FINAIS}

No contexto geral da evasão do ensino superior, é perceptível que no curso de Engenharia Elétrica há um problema grande relacionado às disciplinas envolvendo conhecimentos base de matemática e física. Junto a isso, observar as taxas de reprovação no curso é de suma importância para o ensino acadêmico, uma vez que torna possível elaborar métodos que possam possibilitar a redução das taxas de mais de $50 \%$ de reprovação nas disciplinas iniciais do curso que são possíveis catalisadores dessa evasão. Assim, as ações de elaboração de cursos voltados para disciplinas base da grade curricular são legítimas para nivelar os conhecimentos e apoiar os ingressantes a permanecer no ensino superior.

Dessa forma, o grupo ao contribuir desenvolvendo os cursos anteriormente citados da forma mais objetiva e didática possível, pôde resultar por exemplo, no curso de Cálculo I, em um alcance de 6 cursos diferentes, além das métricas de mais de 3700 visualizações totais em ambos os cursos e respostas de exercícios, demonstrando a eficácia no caminho de auxiliar alunos de graduação em obter um melhor aproveitamento em suas disciplinas cumprindo assim, o objetivo inicial da preparação das aulas.

\section{REFERÊNCIAS}

BARRETO, L. K. S.; FIGUEIREDO, M. F. A.; MEDEIROS, C. M.; ALVES, M. V. P. C. F. Linguagem, comunicação e inclusão: a importância do curso de nivelamento em língua portuguesa para os cursos superiores de tecnologia da Universidade Potiguar - UNP. Revista Connexio, ano 1, v. 2, p. 99-112, 2011.

\section{BLEICHER, Sabrina. A influência dos novos media no design editorial: estudo do} projecto gráfico da Folha de S. Paulo. 2009. 135 f. Dissertação (Mestrado em Estudos Editoriais) - Departamento de Línguas e Culturas, Universidade de Aveiro, Aveiro, 2009. Disponível em: https://ria.ua.pt/bitstream/10773/2820/1/2009001211.pdf.

\section{BOSSA N. Problemas de Aprendizagem: Estudos Revelam que Alunos Acumulam} Defasagem Durante o Ensino Fundamental. $1^{\circ}$ Ed. 2009. Disponível em: http://www.portalguiaescolas.com.br/acontecenasescola s/espacoedzcacional/problemasdeaprendizagemestudor evelaquealunosacumulamdefasagemduranteoensinofundamental-por-vagner-apinhanesi. 
CRISTINA A, BISPO R. Problemas na Educação Prejudicam Alunos na Universidade. $1^{\circ}$ Ed. 2017. Acesso em 18 de Março de 2019. Disponível em: http://codigo.inf.br/aun/educacao/problemasnaeducacaoprejudicam-alunosnauniversidade/

JESUS, R. P. S.; SOUTO, D. P. Cálculo III com o uso de Vídeos na Educação a Distância.

COINSPIRAÇÃO_Revista de Professores que Ensinam Matemática. v. 1 n. 2, jul-dez. 2018. Disponível

em:

http://sbemmatogrosso.com.br/publicacoes/index.php/coinspiracao/article/view/30.

SILVA FILHO, Roberto Leal Lobo et al. A evasão no ensino superior brasileiro. Cad. Pesqui., São Paulo, v. 37, n. 132, p. 641-659, Dez. 2007. Disponível em: $<$ http://www.scielo.br/scielo.php?script=sci arttext\&pid=S0100-

$15742007000300007 \&$ Ing=en\&nrm=iso $>$ acessado em: 16 Abr.
2021. http://dx.doi.org/10.1590/S0100-15742007000300007.

BORTOLANZA, Juarez. Trajetória do Ensino Superior Brasileiro. 2017. Disponível em: https://repositorio.ufsc.br/bitstream/handle/123456789/181204/101 00125.pdf?sequence= 1\&isAllowed $=\mathrm{y}$.

\section{Internet:}

Instituto Nacional de Estudos e Pesquisas Educacionais Anísio Teixeira. Ministério da Educação. Censo da Educação Superior 2010. Disponível em: https://download.inep.gov.br/download/superior/censo/2010/resumo tecnico censo educ acao superior 2010.pdf. Acesso em: 19 maio 2021.

Instituto Nacional de Estudos e Pesquisas Educacionais Anísio Teixeira. Ministério da Educação. Censo da Educação Superior 2019. Disponível em: https://download.inep.gov.br/educacao superior/censo superior/documentos/2020/Notas Estatisticas Censo da Educacao Superior 2019.pdf. Acesso em: 19 maio 2021.

Problema da Evasão no Ensino Superior Brasileiro. Disponível em: https://www.institutolobo.org.br/core/uploads/artigos/art 087.pdf. Acesso em: 19 maio 2021.

7 de cada 10 alunos do ensino médio têm nível insuficiente em português e matemática, diz MEC. Disponível em: https://g1.globo.com/educacao/noticia/2018/08/30/7-de-cada-10-alunos-do-ensino-mediotem-nivel-insuficiente-em-portugues-e-matematica-diz-mec.ghtml. Acesso em: 02 abr. 2021.

\section{Softwares abertos:}

OBS Studio: software livre e de código aberto para gravação de vídeo e transmissão em tempo real. Disponível em: https://obsproject.com/pt-br. Acesso em: 19 maio 2021. 
Openshot: software livre de edição de vídeo. Disponível em: https://www.openshot.org/pt/. Acesso em: 19 maio 2021.

Shotcut: editor de vídeo gratuito de código aberto. Disponível em: https://shotcut.org/. Acesso em: 19 maio 2021.

\title{
EVASION IN THE ELECTRICAL ENGINEERING GRADUATION AND ANALYSIS OF COMPLEMENTARY ACCOMPANIMENT ON CALCULUS AND ELECTRICAL CIRCUITS COURSES
}

\begin{abstract}
Due the relatively high numbers of dropout and failure rates in the disciplines that composes the initial curriculum of the electrical engineering course at the Federal University of Paraíba, there is an urgent need of development of method and materials that may contribute to the minimization of these numbers. Thus, this paper reports a group of students' work that aims to analyze the impacts related to Calculus I and Electrics Circuits I courses that were developed by the group in order to be a complementary support for the graduation students.
\end{abstract}

Keywords: Dropout. Graduation. Engineering. 